\title{
The Functional SOCS3 RS115785973 Variant Regulated by MiR-4308 Promotes Gastric Cancer Development in Chinese Population
}

\author{
Xiaowei Wang ${ }^{\mathrm{a}}$ Ting $\mathrm{Li}^{\mathrm{a}}$ Meng $\mathrm{Li}^{\mathrm{a}} \mathrm{NaCao^{ \textrm {b } }}$ Jun Han \\ aDepartment of Clinical laboratory, Nanjing Children's Hospital Affiliated to Nanjing Medical University, \\ Nanjing, 'Department of Clinical laboratory, Nanjing Central Hospital, Nanjing, China
}

\section{Key Words}

SOCS3 • miR-4308 • 3'-UTR • Gastric cancer • Polymorphism

\begin{abstract}
Background/Aims: SOCS3 is tumor suppressor which has been identified as upstream of JAK/STAT3 signaling by specific kinase inhibition. However, additional regulations especially through a non-coding RNA approach were remained unknown. Methods: We performed casecontrol study focusing on the miRNAs associated SNPs in SOCS3 to investigate the further relationship of the SNPs with miRNAs among Chinese gastric cancer (GC) patients. Genotyping, real time PCR assay, cell transfection and the dual luciferase reporter assay were used in our study. Results: We found that patients suffering from Helicobacter pylori $(H$. pylori) infection indicating as susceptible population by comparing with controls. Besides, SNP rs 115785973 in SOCS3 was identified as a risk factor in the occurrence of GC highly associated with poor differentiation grade, larger tumor size and metastasis. In vitro assay found that rs 115785973 could be regulated by miR-4308 which caused an up-regulation of SOCS3 in patients with GA and AA genotype. Conclusion: Our findings have shown that the SNP rs115785973 in SOCS3 disrupting the regulatory role of miR-4308 in SOCS3 expression, rs115785973 in SOCS3 might act as a risk factor in the pathogenesis of GC.
\end{abstract}

(C) 2016 The Author(s)

Published by S. Karger AG, Basel

\section{Introduction}

Gastric cancer (GC) is one of the most common malignancies and remains an important cause of mortality worldwide, especially in East Asia (e.g., China, Japan, and South Korea). About $42 \%$ of worldwide cases occur in China. Currently, gastric carcinoma prognosis is primarily determined based on the clinical data and pathologic stages of patients at the time of diagnosis and treatment [1]. The recent surgery, chemotherapeutic, immunologic, and radiation approaches have provided a significant improvement for the survival of patients 
with localized disease; however, successful management of patients with gastric carcinoma is still hampered by the lack of highly sensitive and specific biomarkers capable of predicting prognosis and likelihood of metastasis [2-4]. Etiological studies have suggested that environmental factors, including diet high in salted and nitrated foods, tobacco use, alcoholic consumptions as well as Helicobacter pylori (H. pylori) infection was highly associated with GC; However, only a fraction of people adopting exposed to the same environmental risk factors eventually developed gastric cancer, suggesting that genetic factors may also play a role in the etiology of the disease [5-7].

SOCS family proteins form part of a classical negative feedback system that regulates cytokine signal transduction. SOCS3 is involved in negative regulation of cytokines that signal through the JAK/STAT pathway $[6,8]$ and could inhibit cytokine signal transduction by binding to tyrosine kinase receptors including gp130, LIF, erythropoietin, insulin, IL12, GCSF and leptin receptors. SOCS3 was also documented as a potential indicator for prediction the lymph node metastasis from GC [9]. Besides, low expression of SOCS1 and SOCS3 was considered as a poor prognostic indicator for gastric cancer patients [10]. Several studies indicated that SOCS3 could be regulated by special miRNA [11]. For example, miR-185 can directly target SOCS3 by binding to its 3'-untranslated region (UTR) thus lead to enhanced insulin secretion of pancreatic $\beta$-cells, promoted cell proliferation and protected cells from apoptosis [12]. And miR-19b suppressed the inflammatory response by inhibiting SOCS3 to modulate chemokine production in intestinal epithelial cells (IECs) and thereby prevents the pathogenesis of crohn disease [13], but the shift regulation caused by polymorphism within 3'UTR of SOCS3 have never been touched.

Thus, in this study, we focused on the SNPs in the 3'UTR of SOCS3. By using the bioinformatics software (http://www.bioguo.org/miRNASNP/), we obtained all the SNPs which could regulate by miRNAs as candidate SNPs. Via the bioinformatics prediction, we found the rs115785973 was the potential SNP which might potential regulated by miR-4308 and we further investigated the allele distribution in a case-control study.

\section{Materials and Methods}

Study subjects

A total of 722 GC cases from Nanjing Central Hospital and Municipal authorities Hospital as well as 693 sex- and age-matched controls were enrolled in this study. Patients were consecutively recruited between February 2010 and January 2015 at both Hospitals. The diagnosis of all patients was histological confirmed. A face-to-face questionnaire was administered to collect demographic data and environmental exposure information, including H. pylori infection tumor differentatation, tumor size and metastasis. All participants have provided their written informed consents to participate in this study. The detailed information of patients has been presented in Table 1. This study was approved by the institutional Review Board of Nanjing Children's Hospital and Municipal authorities Hospital.

Allele distribution detection

The polymorphism was genotyped through the probe based polymerase chain reaction (PCR) detection as described previously [14]. The PCR reactions were carried out in a total volume of $5 \mu \mathrm{L}$ containing TaqMan Universal Master Mix, 80X SNP Genotyping AssayMix, Dnase-free water and 10 ng genomic DNA. The PCR conditions were $2 \mathrm{~min}$ at $50^{\circ} \mathrm{C}, 10 \mathrm{~min}$ at $95^{\circ} \mathrm{C}$, followed by 40 cycles at $95^{\circ} \mathrm{C}$ for $15 \mathrm{sec}$ and $60^{\circ} \mathrm{C}$ for $1 \mathrm{~min}$. The 384-well ABI 7900HT Real Time PCR System. A 10\% random sample was reciprocally examined by different persons, and the reproducibility was $100 \%$.

Real time PCR assay

Real time polymerase chain reaction (PCR) was performed to determine whether the G to A mutation changed the expression level of SOCS3. Total RNA was obtained from tissues using TRIzol reagent as described by the manufacturer (Invitrogen Life Technologies Co, CA, USA) and was qualified with the 260/280nm absorbance ratio (1.8 - 2.0). For mRNA detection, total RNAs (500 ng) were reversed transcribed using 


\section{Cellular Physiology Cell Physiol Biochem 2016;38:1796-1802 \begin{tabular}{l|l} 
and Biochemistry 10.1159/000443118 & $\begin{array}{l}\text { DO 2016 The Author(s). Published by S. Karger AG, Basel } \\
\text { Pww.karger.com/cpb }\end{array}$ \\
\hline
\end{tabular} \\ Wang et al.: MiR-4308 Associated SNP in SOCS3}

the reverse transcription kit (Takara, Tokyo, Japan). GAPDH was used as an internal control. The amplification conditions were $95^{\circ} \mathrm{C}$ for 10 minutes, followed by 40 cycles of $95^{\circ} \mathrm{C}$ for 30 seconds, $55^{\circ} \mathrm{C}$ for 40 seconds, and $72^{\circ} \mathrm{C}$ for 30 seconds, and finally $4^{\circ} \mathrm{C}$ for 30 minutes for cooling as described. Real time PCR was performed using ABI Prism 7900HT (Applied Biosystems, CA, USA) according to the direction of the reagents.

\section{Cell lines and cell culture}

GC cell lines SGC-7901 and MKN-45 were purchased from the Chinese Academy of Sciences Cell Bank (Shanghai, China). All cells were cultured in Dulbecco's Minimum Essential Medium(DMEM) purchased from Gibco (CA, USA) supplemented with $10 \%$ fetal bovine serum (Invitrogen, Carlsbad, USA) and grown in humidified $5 \% \mathrm{CO} 2$ at $37^{\circ} \mathrm{C}$. MiR4308 mimics and normal control were obtained from Genepharma (Shanghai,
Table 1. Frequency distributions of selected variables in patients and cancer-free controls. ${ }^{*}$ Two-sided chi-square test for either genotype distributions or allele frequencies between cases and controls

\begin{tabular}{|c|c|c|c|c|c|}
\hline \multirow{2}{*}{ Variables } & \multicolumn{2}{|c|}{ Cases $(n=722)$} & \multicolumn{2}{|c|}{ Controls $(n=693)$} & \multirow{2}{*}{$P$} \\
\hline & $N$ & $\%$ & $N$ & $\%$ & \\
\hline Age (years) & & & & & 0.3882 \\
\hline$\leq 50$ & 376 & $52.08 \%$ & 345 & $49.78 \%$ & \\
\hline$>50$ & 346 & $47.92 \%$ & 348 & $50.22 \%$ & \\
\hline Gender & & & & & 0.4525 \\
\hline Male & 318 & $44.04 \%$ & 319 & $46.03 \%$ & \\
\hline Female & 404 & $55.96 \%$ & 374 & $53.97 \%$ & \\
\hline H. pylori & & & & & 0.0007 \\
\hline Negative & 254 & $35.18 \%$ & 186 & $26.84 \%$ & \\
\hline Positive & 468 & $64.82 \%$ & 507 & $73.16 \%$ & \\
\hline \multicolumn{6}{|c|}{ Differentiation grade } \\
\hline Well & 276 & $38.23 \%$ & & & \\
\hline Moderate & 251 & $34.76 \%$ & & & \\
\hline Poorly & 195 & $27.01 \%$ & & & \\
\hline \multicolumn{6}{|c|}{ Tumor Size $(\mathrm{cm})$} \\
\hline$\leq 3 \mathrm{~cm}$ & 412 & $57.06 \%$ & & & \\
\hline$>3 \mathrm{~cm}$ & 310 & $42.94 \%$ & & & \\
\hline \multicolumn{6}{|l|}{ Metastasis } \\
\hline Yes & 287 & $39.75 \%$ & & & \\
\hline No & 435 & $60.25 \%$ & & & \\
\hline
\end{tabular}

China).The transfection was conducted by using Lipofectamine 2000 (Invitrogen Corp, CA, USA).

\section{Prediction of miRNAs binding to the SNP}

Based on our bioinformatics analysis by using the bioinformatics software (http://www.bioguo.org/ miRNASNP/) to predict the related SNPs in the 3'UTR of SOCS3 which could interact with miRNAs.

Construction of luciferase-based reporter plasmids

A full length fragment of the 3'UTR containing either G or A allele of rs115785973 was amplified. The PCR production was cloned into the pGL3-promoterless luciferase-based plasmid (Promega) at the cloning site between KpnI and XhoI. The amplified fragment was verified by DNA sequencing.

Dual-luciferase reporter assay

For luciferase activity analysis, SGC-7901 and MKN-45 cells were co-transfected with 100 ng of luciferase reporter constructs $5 \mathrm{ng}$ of the $\beta$-gal control plasmid and 10 pmol of miRNAs with $1 \mu$ lipofectamine 2000 according to the manufacturer's instructions (Invitrogen, NY, USA). After incubation for 48h, we carried out the luciferase assay using the luciferase reporter assay system (Promega, Madison, WI) according to the manufacturer's protocol. Measurements of luminescence and absorbance of $\beta$-gal were performed on a luminometer (Glomax 20/20; Promega). Three independent experiments were performed in triplicate.

\section{Statistical analysis}

Differences between cases and controls were evaluated by the Student's t-test for continuous variables and the $\mathrm{x}^{2}$ test for categorical variables. The association between SNPs and GC risk was estimated by the OR and 95\% confidence interval (CI) using the general genetic model. The potential gene-environment interaction was evaluated by logistic regression analysis and tested by comparing changes in deviance between the models of main effects with or without the interaction term.. Comparisons between groups were analyzed by the t test (two-sided). All statistical analyses were performed using Statistical Package for Social Sciences software. 


\section{Cellular Physiology Cell Physiol Biochem 2016;38:1796-1802 \begin{tabular}{l|l} 
DOI: 10.1159/000443118 & $\begin{array}{l}\text { O 2016 The Author(s). Published by S. Karger AG, Basel } \\
\text { www.karger.com/cpb }\end{array}$
\end{tabular} \\ Wang et al.: MiR-4308 Associated SNP in SOCS3}

\section{Results}

The differences in the distribution of the selected variables among GC cases and controls are shown on Table 1 . No significant differences of age $(P=0.3882)$ and sex $(P=0.4525)$ were found between the case and the control group. There were more patients who had $H$. pylori infection in the cases than in the controls $(\mathrm{P}=0.0007)$.

In order to investigate the miRNA associated SNPs in the 3'UTR of SOCS3, we first found all the SNPs from the SNP databases NCBI db SNP BUILED 129 and ENSEMBL v58 in the 3'UTR of SOCS3 gene with the minor allele frequency(MAF) $>0.05$. We then used bioinformatics software Diana-Micro and RNA hybrid to predict miRNAs that can bind to the SOCS3 3'-UTR. The miRNA SNP database was also applied to explore the miRNAs which could also bind to the 3'-UTR of patients harbored the SNP. As was shown in Table 2, we obtained 3 SNPs in 3'UTR of SOCS3 which could be regulated by different miRNAs. The positions of the SNPs in 3'UTR of SOCS3 as well as the variants were listed. Further genotyping was performed to detect the distribution of allele gene of the SNP in our research which is rs115785973 which can be potentially regulated by miR-4308. The results showed that the genotypes of rs115785973 were in Hardy-Weinberg equilibrium distribution pattern in the healthy control group $(\mathrm{P}<0.0001)$.

Logistic regression analyses indicated that individuals with the GA and AA genotype of rs115785973 in 3'-UTR of SOCS3 was significantly associated with GC risk (P < 0.0001). Moreover, the significant association with GC risk was shown in A carrier $(\mathrm{P}<0.0001)$ (Table 3 ). Besides, patients with allele A in 3'UTR of SOCS3 were significantly associated GC risk, especially AA genotype of in 3'UTR of SOCS3, (for AA: OR $=2.21,95 \% \mathrm{CI}=1.27-1.79$; for GA: $\mathrm{OR}=1.87,95 \% \mathrm{CI}=1.53-1.96$; and for A carrier: $\mathrm{OR}=2.03 ; 95 \% \mathrm{CI}=1.55-1.98)$. All ORs were adjusted for sex, age, and H. pylori infection.

Then, we conducted stratified analysis of the association of the rs115785973 and with the clinicopathological parameters of GC (Table 4). We found a significant association of the rs113054794 genotypes with the tumor size, differentiation and metastasis. Compared with the CC homozygote, the carriers of A allele presented significant large tumor size, poor differentiation as well as the high potential of metastasis.

Table 2. Candidate SNPs in the 3'UTR of SOCS3. Chr = chromosome; UTR= Untranslated Regions

\begin{tabular}{lcccc}
\multicolumn{1}{c}{ SNP } & Chr & $\begin{array}{c}\text { 3'UTR } \\
\text { position }\end{array}$ & $\begin{array}{c}\text { Associated } \\
\text { miRNA }\end{array}$ & Allele* \\
\hline SOCS3 & & & & \\
rs72899753 & 17 & $14-36$ & hsa-miR-3183 & G/A \\
rs115785973 & 17 & $533-550$ & hsa-miR-4308 & G/A \\
rs185147690 & 17 & $527-549$ & hsa-miR-4254 & G/A \\
\hline
\end{tabular}

Table 3. Genotype frequencies of the SOCS3 at rs115785973 polymorphism among GC cases and controls. aThe ORs, 95\%CIs and P value were calculated after adjusting for age, gender, parental H. pylori infection history and family cancer history

\begin{tabular}{|c|c|c|c|c|c|c|}
\hline \multirow{2}{*}{ Genotype } & \multicolumn{2}{|c|}{ Cases $(n=722)$} & \multicolumn{2}{|c|}{ Controls $(n=693)$} & \multirow[t]{2}{*}{$\begin{array}{c}\text { OR } \\
(95 \% \mathrm{CI})^{\mathrm{a}}\end{array}$} & \multirow[t]{2}{*}{$P$ Value } \\
\hline & $N$ & $\%$ & $N$ & $\%$ & & \\
\hline \multicolumn{7}{|c|}{ rs115785973 } \\
\hline GG & 291 & $40.30 \%$ & 401 & $57.86 \%$ & 1.00 & $<0.0001$ \\
\hline GA & 208 & $28.81 \%$ & 153 & $22.08 \%$ & $1.87(1.53-1.96)$ & \\
\hline AA & 223 & $30.89 \%$ & 139 & $20.06 \%$ & $2.21(1.27-1.79)$ & \\
\hline ACarrier & 431 & $59.70 \%$ & 292 & $42.14 \%$ & $2.03(1.55-1.98)$ & $<0.0001$ \\
\hline
\end{tabular}


By using real-time PCR, we observed that there was significant difference in the SOCS3 expression levels of the patients carrying GG, GA and AA genotype (Fig. 1A). The expression levels of the GA carriers and the AA carriers were significantly lower than those of GG $(\mathrm{P}<0.01)$.

In order to investigate whether the rs115785973 polymorphism affects the predicted miRNAs (miR-4308) combining with the 3'-UTR conservative sites of SOCS3 mRNA, we performed the transient transfection in vitro and measured the relative activities with a Dual-Glo Luciferase Reporter Assay System; the detailed sequence was presented in Fig. 1B. As a result, co-transfection of the luciferase vector with the 3'UTR of SOCS3 containing the
Table 4. Stratified analysis of genotypes of SOCS3 rs115785973 with clinicopathological parameters of GC. *Two-sided chi-square test for either genotype distributions or allele frequencies between cases and controls

\begin{tabular}{lccccc} 
Feather & \multicolumn{5}{c}{ Genotype } \\
& GG & GA & AA & $\begin{array}{c}\text { GA vs GG } \\
P \text { Value* }\end{array}$ & $\begin{array}{c}\text { AA vs GG } \\
P \text { Value* }\end{array}$ \\
\hline Age (years) & & & & & \\
$\leq 50$ & 21 & 27 & 41 & 0.2157 & 0.7408 \\
$>50$ & 26 & 20 & 45 & & \\
Gender & & & & & \\
Male & 24 & 29 & 51 & 0.295 & 0.36 \\
Female & 23 & 18 & 35 & & \\
Differentiation grade & & & & & \\
Well & 33 & 9 & 21 & $<0.0001$ & $<0.0001$ \\
Moderate & 12 & 15 & 27 & & \\
Poorly & 2 & 23 & 38 & & \\
Tumor Size(cm) & & & & & \\
$\leq 5 \mathrm{~cm}$ & 34 & 17 & 38 & 0.0004 & 0.0018 \\
$>5 \mathrm{~cm}$ & 13 & 30 & 48 & & \\
Metastasis & & & & & \\
Yes & 12 & 31 & 57 & $<0.0001$ & $<0.0001$ \\
No & 35 & 16 & 29 & & \\
\hline
\end{tabular}

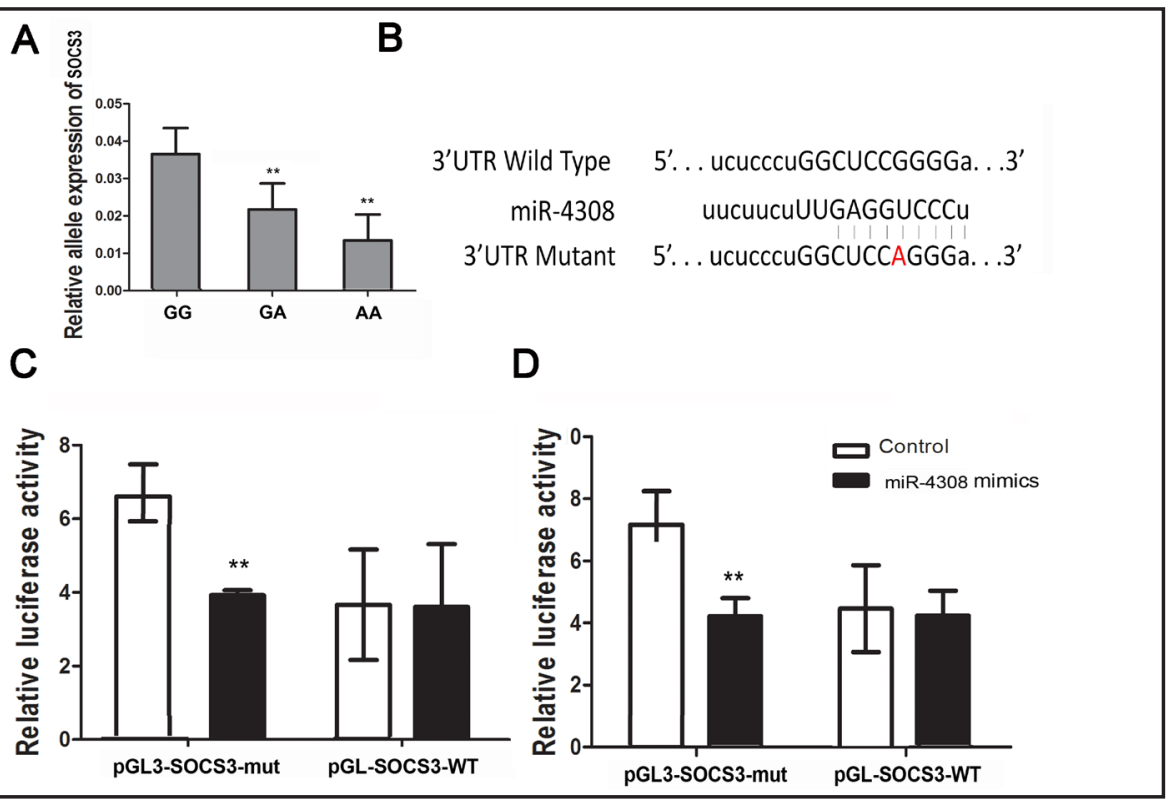

Fig. 1. SNP rs115785973 in 3'UTR of GC patients with A allele can down-regulate SOCS3 transcription by deregulating of miR-4083. (A) The expression level of SOCS3 was determined by real time PCR in patients with GG, GA and AA genotypes. (B) Bioinformatics predicted the binding site between the miR-4308 with SOCS3 and the mutation type were conducted into the pGL3 plasmid as presented. (C) Cells were co-transfected with miR-4308 mimics or control, Renilla luciferase vector pRL-SV40 for $48 \mathrm{~h}$. Both firefly and Renilla luciferase activities were measured in the same sample. Firefly luciferase signals were normalized with Renilla luciferase signals. Left panel indicated the SGC-7901 cell line while the right indicated MNK-45 cell lines. Data was presented as the mean \pm SEM. * indicates a significant difference $(\mathrm{P}<0.05)$. 
mutated allele A and miR-4308 into two cells significantly changed the luciferase expression level compared with the reporter plasmid containing the wild genotype (Fig. 1C, D). Thus, we concluded that the polymorphism affected binding ability of miR-4308 with 3'UTR of SOCS3 mRNA and then affect the function of post-transcriptional regulation, resulting in the abnormal expression level of SOCS3.

\section{Discussion}

SOCS3 is a tumor suppressor which is involved in negative regulation of cytokines that signal through the JAK/STAT pathway. So far, there are many reports revealed that SOCS3 was down-regulated and acted as tumor suppressor in many tumors including liver cancer, colorectal cancer, breast cancer and cholangiocarcinoma $[15,16]$. Ohara K showed that gastrointestinal epithelial cell-specific SOCS3 conditional knockout (T3b-SOCS3 cKO) mice developed gastric tumors by enhancing leptin production and the ObRb/signal transducer and activator of transcription 3 (STAT3) signaling pathway. These tumors displayed pathology and molecular alterations, such as an increase in MUC2 (Mucin 2, oligomeric mucus/gel-forming) and TFF3 (trefoil factor 3), resembling human intestinal-type gastric tumors [17]. In addition, low SOCS3 expression are poor prognostic indicators in human GC, and Deng et al. revealed that the SOCS3 was the best predicator of lymph node metastasis from GC identified with the nominal regression analysis $[9,10]$. Therefore, SOCS3 might be considered as a potential indicator for prediction the lymph node metastasis from GC. All above finding indicated that SOCS3 was a suppressor in gastric cancer development and metastasis which was similar to our findings that gain SNP of 3'-UTR of SOCS3 by miR-4308 can suppress its expression, thus to increase incidence of tumorigenesis of gastric cancer.

MiRNAs can not only negatively regulate their target genes expression at the posttranscription level through binding to 3'-UTR of their targets message RNAs [18-20], but also can regulate the 3'-UTR region who harboured the single-nucleotide polymorphisms $[21,22]$. The SNPs of 3'-UTRs region of a certain gene can potentially regulate the impact of microRNA on post-transcriptional regulation and its potential relation to complex diseases. More and more studies have provided evidence that SNPs located in the miRNA (miRSNPs) binding sites through affecting the binding of miRNAs with the target genes resulted in decrease or increase in the target mRNA translation, and thus being associated with the susceptibility to cancers [23-25]. In present study, the predicted SNP of rs115785973 in 3'UTR region of SOCS3 could affect the binding efficiency of miR-4308. Moreover, increased expression of SOCS3 were detected which might indicated that the decreased suppressive effect from miR-4308 and can served as another reason of increased SOCS3 in human GC.

In conclusion, our findings demonstrated that the SNP rs115785973 located in the 3'-UTR of SOCS3 was highly associated with tumor differentiation, tumor growth and metastasis. This SNP could also regulated by miR-4308 resulting in an abnormal SOCS3 expression, rs115785973 in SOCS3 might act as a risk factor in the pathogenesis of GC.

\section{Disclosure Statement}

None.

\section{References}

1 Pourhoseingholi MA, Vahedi M, Baghestani AR: Burden of gastrointestinal cancer in Asia; an overview. Gastroenterol Hepatol Bed Bench 2015;8:19-27.

2 Amieva M, Peek RM, Jr.: Pathobiology of Helicobacter pylori-induced Gastric Cancer. Gastroenterology 2016;150:64-78. 


\section{Cellular Physiology Cell Physiol Biochem 2016;38:1796-1802 \begin{tabular}{l|l} 
DOI: 10.1159/000443118 & $\begin{array}{l}\text { O 2016 The Author(s). Published by S. Karger AG, Basel } \\
\text { www.karger.com/cpb }\end{array}$
\end{tabular} \\ Wang et al.: MiR-4308 Associated SNP in SOCS3}

3 Zhu P, Zhang J, Zhu J, Shi J, Zhu Q, Gao Y: MiR-429 Induces Gastric Carcinoma Cell Apoptosis Through Bcl-2. Cell Physiol Biochem 2015;37:1572-1580.

4 Liu Y, Xu J, Jiang M, Ni L, Chen Y, Ling Y: Association between functional PSMD10 Rs111638916 variant regulated by MiR-505 and gastric cancer risk in a Chinese population. Cell Physiol Biochem 2015;37:10101017.

5 Katoh M, Katoh M: STAT3-induced WNT5A signaling loop in embryonic stem cells, adult normal tissues, chronic persistent inflammation, rheumatoid arthritis and cancer (Review). Int J Mol Med 2007;19:273278.

6 Milne AN, Carneiro F, O'Morain C, Offerhaus GJ: Nature meets nurture: molecular genetics of gastric cancer. Hum Genet 2009;126:615-628.

7 Wang D, Fan Z, Liu F, Zuo J: Hsa-miR-21 and Hsa-miR-29 in Tissue as Potential Diagnostic and Prognostic Biomarkers for Gastric Cancer. Cell Physiol Biochem 2015;37:1454-1462.

8 Linossi EM, Nicholson SE: Kinase inhibition, competitive binding and proteasomal degradation: resolving the molecular function of the suppressor of cytokine signaling (SOCS) proteins. Immunol Rev 2015;266:123-133.

9 Deng J, Jiao X, Liu H, Wu L, Zhang R, Wang B, Pan Y, Hao X, Liang H: Lymph node metastasis is mediated by suppressor of cytokine signaling-3 in gastric cancer. Tumour Biol 2013;34:3627-3636.

10 Li G, Xu J, Wang Z, Yuan Y, Li Y, Cai S, He Y: Low expression of SOCS-1 and SOCS-3 is a poor prognostic indicator for gastric cancer patients. J Cancer Res Clin Oncol 2015;141:443-452.

11 Woo JH, Lee JH, Kim H, Park SJ, Joe EH, Jou I: Control of Inflammatory Responses: a New Paradigm for the Treatment of Chronic Neuronal Diseases. Exp Neurobiol 2015;24:95-102.

12 Bao L, Fu X, Si M, Wang Y, Ma R, Ren X, Lv H: MicroRNA-185 targets SOCS3 to inhibit beta-cell dysfunction in diabetes. PLoS One 2015;10:e0116067.

13 Cheng X, Zhang X, Su J, Zhang Y, Zhou W, Zhou J, Wang C, Liang H, Chen X, Shi R, Zen K, Zhang CY, Zhang H: miR-19b downregulates intestinal SOCS3 to reduce intestinal inflammation in Crohn's disease. Sci Rep 2015;5:10397.

14 Yan R, Yang J, Jiang P, Jin L, Ma J, Huang R, Ma N, Jiang F: Genetic variations in the SOCS3 gene in patients with Graves' ophthalmopathy. J Clin Pathol 2015;68:448-452.

15 Inagaki-Ohara K, Kondo T, Ito M, Yoshimura A: SOCS, inflammation, and cancer. JAKSTAT 2013;2:e24053.

16 Yin Y, Liu W, Dai Y: SOCS3 and its role in associated diseases. Hum Immunol 2015;76:775-780.

17 Inagaki-Ohara K, Mayuzumi H, Kato S, Minokoshi Y, Otsubo T, Kawamura YI, Dohi T, Matsuzaki G, Yoshimura A: Enhancement of leptin receptor signaling by SOCS3 deficiency induces development of gastric tumors in mice. Oncogene 2014;33:74-84.

18 Valastyan S, Reinhardt F, Benaich N, Calogrias D, Szasz AM, Wang ZC, Brock JE, Richardson AL, Weinberg RA: A pleiotropically acting microRNA, miR-31, inhibits breast cancer metastasis. Cell 2009;137:10321046.

19 Hyun S, Lee JH, Jin H, Nam J, Namkoong B, Lee G, Chung J, Kim VN: Conserved MicroRNA miR-8/miR-200 and its target USH/FOG2 control growth by regulating PI3K. Cell 2009;139:1096-1108.

20 Zhou X, Ye F, Yin C, Zhuang Y, Yue G, Zhang G: The Interaction Between MiR-141 and IncRNA-H19 in Regulating Cell Proliferation and Migration in Gastric Cancer. Cell Physiol Biochem 2015;36:1440-1452.

21 Wang K, Diskin SJ, Zhang H, Attiyeh EF, Winter C, Hou C, Schnepp RW, Diamond M, Bosse K, Mayes PA, Glessner J, Kim C, Frackelton E, Garris M, Wang Q, Glaberson W, Chiavacci R, Nguyen L, Jagannathan J, Saeki N, Sasaki H, Grant SF, Iolascon A, Mosse YP, Cole KA, Li H, Devoto M, McGrady PW, London WB, Capasso M, Rahman N, Hakonarson H, Maris JM: Integrative genomics identifies LMO1 as a neuroblastoma oncogene. Nature 2011;469:216-220.

22 Wang QX, Zhu YQ Zhang H, Xiao J: Altered MiRNA expression in gastric cancer: a systematic review and meta-analysis. Cell Physiol Biochem 2015;35:933-944.

23 Bartel DP: MicroRNAs: genomics, biogenesis, mechanism, and function. Cell 2004;116:281-297.

24 Wang Y, Zhou L, Chen J, Li J, He L, Wu P, Wang M, Tong N, Zhang Z, Fang Y: Association of the 3'UTR FOXO3a polymorphism rs4946936 with an increased risk of childhood acute lymphoblastic leukemia in a Chinese population. Cell Physiol Biochem 2014;34:325-332.

25 Chen Q, Qin R, Fang Y, Li H, Liu Y: A Functional Variant at the miR-214 Binding Site in the Methylenetetrahydrofolatereductase Gene Alters Susceptibility to Gastric Cancer in a Chinese Han Population. Cell Physiol Biochem 2015;36:622-630. 